Homology, Homotopy and Applications, vol. 9(1), 2007, pp.139-161

\title{
3-TYPES OF SIMPLICIAL GROUPS AND BRAIDED REGULAR CROSSED MODULES
}

\author{
Z. ARVASI AND E. ULUALAN
}

(communicated by Ulf Rehmann)

\begin{abstract}
In this work, we explain the relations among braided regular crossed modules, simplicial groups, 2-crossed modules, quadratic modules and crossed squares, and the role of hypercrossed complex pairings in these structures.
\end{abstract}

\section{Introduction}

As an algebraic model of connected (weak homotopy) 3-types, the notion of 2crossed module was introduced by Conduché in [16], and these 2-crossed modules are equivalent to simplicial groups with Moore complex of length 2. Crossed squares and quadratic modules are other algebraic models of connected 3-types defined by Loday and Guin-Walery [26] and Baues [6] respectively. In [5], we explored the relations among 2-crossed modules, quadratic modules, crossed squares and simplicial groups, and the homotopy equivalences between these structures.

Brown and Gilbert in [9] introduced the notion of braided, regular crossed module as an alternative algebraic model of homotopy 3-types. They then showed that this structure is closely related to simplicial groups; they proved that the category of braided, regular crossed modules is equivalent to that of simplicial groups with Moore complex of length 2. They have also proved that braided, regular crossed modules are equivalent to Conduché's 2-crossed modules.

Related ideas of Conduché have been used by Carrasco and Cegarra [14] to study braided categorical groups (see also Garzon and Miranda [20]). Carrasco and Cegarra [13] defined the notion of $n$-hypercrossed complex as an algebraic model of connected $(n+1)$-types. The article [4] is one of a series in which the first author and Porter studied the higher dimensional Peiffer elements, called hypercrossed complex pairings $F_{\alpha, \beta}$, by using ideas of Conduché (cf. [16]) and techniques developed by Carrasco and Cegarra (cf. [13]), and they applied their results in various homological settings and then gave a reformulation of Conduché's result in terms of hypercrossed complex pairings for commutative algebras. Mutlu and Porter [24] have also adapted their method to simplicial groups. Castiglioni and Ladra $[\mathbf{1 5}]$ generalized the results proved in $[\mathbf{1}],[\mathbf{4}]$ and $[\mathbf{2 4}]$.

Received August 10, 2006, revised October 18, 2006; published on January 12, 2007.

2000 Mathematics Subject Classification: 18D35, 18G30, 18G50, 18 G55.

Key words and phrases: 2-crossed modules, simplicial groups, braided regular crossed modules.

Copyright (C) 2007, International Press. Permission to copy for private use granted. 
In the present article, we explain the relations among braided regular crossed modules and algebraic models of connected (weak homotopy) 3-types mentioned above.

Thus, the main points of this paper are:

(i) to give the relation between braided regular crossed modules and simplicial groups in terms of hypercrossed complex pairings $F_{\alpha, \beta}$ defined in [24],

(ii) to give the connection between crossed squares and braided regular crossed modules by using the relation between 2-crossed modules and crossed squares as described in [5, Section 4] in terms of bisimplicial nerve of the crossed square,

(iii) to give a construction of a quadratic module from a braided regular crossed module, by using the construction of a quadratic module from a 2-crossed module as given in [5, Section 5].

Therefore, the results of this paper can be summarized in the following diagram

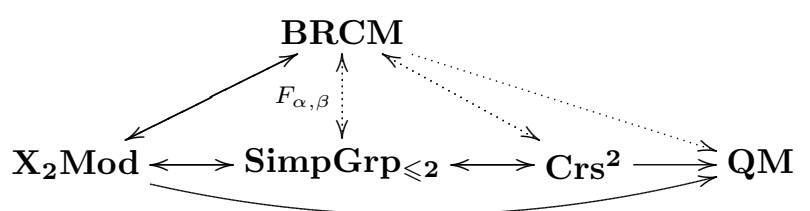

where the diagram is commutative, linking the broken arrows given below and the unbroken arrows given in [5].

\section{Preliminaries}

\section{Simplicial groups}

Denoting the usual category of finite ordinals by $\Delta$, we obtain for each $k \geqslant 0$ a subcategory $\Delta_{\leqslant k}$ determined by the objects $[j]$ of $\Delta$ with $j \leqslant k$. A simplicial group is a (contravariant) functor from the opposite category $\Delta^{o p}$ to the category of groups Grp. A reduced simplicial group is a simplicial group whose first component is trivial. A $k$-truncated simplicial group is a functor from $\Delta_{\leqslant k}^{o p}$ to Grp. We will denote the category of simplicial groups by SimpGrp and the category of $k$-truncated simplicial groups by $\operatorname{Tr}_{k}$ SimpGrp. By a $k$-truncation of a simplicial group, we mean a $k$-truncated simplicial group $\operatorname{tr}_{k} \mathbf{G}$ obtained by forgetting dimensions of order $>k$ in a simplicial group $\mathbf{G}$. This gives a truncation functor $\mathbf{t r}_{k}: \mathbf{S i m p G r p}_{\mathbf{S i m}} \rightarrow$ $\operatorname{Tr}_{k}$ SimpGrp which admits a right adjoint $\operatorname{cosk}_{k}: \operatorname{Tr}_{k} \operatorname{SimpGrp} \rightarrow \operatorname{SimpGrp}$ called the $k$-coskeleton functor, and a left adjoint $\mathbf{s k}_{k}: \operatorname{Tr}_{k} \operatorname{SimpGrp} \rightarrow \operatorname{SimpGrp}$, called the $k$-skeleton functor. For explicit constructions of these, see [18].

Given a simplicial group $\mathbf{G}$, the Moore complex $(\mathbf{N G}, \partial)$ of $\mathbf{G}$ is the normal chain complex defined by

$$
(N G)_{n}=\bigcap_{i=0}^{n-1} \operatorname{ker} d_{i}^{n}
$$

with $\partial_{n}: N G_{n} \rightarrow N G_{n-1}$ induced from $d_{n}^{n}$ by restriction. 
The $n^{\text {th }}$ homotopy group $\pi_{n}(\mathbf{G})$ of $\mathbf{G}$ is the $n^{\text {th }}$ homology of the Moore complex of $\mathbf{G}$, i.e.

$$
\pi_{n}(\mathbf{G}) \cong H_{n}(\mathbf{N G}, \partial)=\bigcap_{i=0}^{n} \operatorname{ker} d_{i}^{n} / d_{n+1}^{n+1}\left(\bigcap_{i=0}^{n} \operatorname{ker} d_{i}^{n+1}\right)
$$

We say that the Moore complex NG of a simplicial group $\mathbf{G}$ is of length $k$ if $N G_{n}=1$ for all $n \geqslant k+1$, so that a Moore complex of length $k$ is also of length $l$ for $l \geqslant k$. We denote the category of simplicial groups with Moore complex of length $k$ by $\operatorname{SimpGrp}_{\leqslant k}$.

The following lemma is due to Conduché [16].

Lemma 1.1. Let $\mathbf{G}$ be a simplicial group. The Moore complex of its $k$-coskeleton $\operatorname{Cosk}_{\mathbf{k}}\left(\operatorname{tr}_{\mathbf{k}}(\mathbf{G})\right)$ is of length $k+1$, i.e.,

$$
N\left(\operatorname{Cosk}_{\mathbf{k}}\left(\operatorname{tr}_{\mathbf{k}}(\mathbf{G})\right)\right)_{i}=1 \text { for } i>k+1,
$$

and is identical to the Moore complex of $\mathbf{G}$ in dimension less than $k+1$. Moreover

$$
N\left(\operatorname{Cosk}_{\mathbf{k}}\left(\operatorname{tr}_{\mathbf{k}}(\mathbf{G})\right)\right)_{k+1}=\operatorname{ker}\left(\partial_{k}: N G_{k} \rightarrow N G_{k-1}\right)
$$

and the morphism $\partial_{k+1}: N\left(\operatorname{Cosk}_{\mathbf{k}}\left(\mathbf{t r}_{\mathbf{k}}(\mathbf{G})\right)\right)_{k+1} \rightarrow N\left(\mathbf{C o s k}_{\mathbf{k}}\left(\mathbf{t r}_{\mathbf{k}}(\mathbf{G})\right)\right)_{k}=N G_{k}$ is injective.

\section{The poset of surjective maps}

The following notation and terminology is derived from [12] and published version [13].

For the ordered set $[n]=\{0<1<\cdots<n\}$, let $\alpha_{i}^{n}:[n+1] \rightarrow[n]$ be the increasing surjective map given by;

$$
\alpha_{i}^{n}(j)= \begin{cases}j & \text { if } j \leqslant i \\ j-1 & \text { if } j>i\end{cases}
$$

Let $S(n, n-r)$ be the set of all monotone increasing surjective maps from $[n]$ to $[n-r]$. This can be generated from the various $\alpha_{i}^{n}$ by composition. The composition of these generating maps is subject to the following rule: $\alpha_{j} \alpha_{i}=\alpha_{i-1} \alpha_{j}, j<i$. This implies that every element $\alpha \in S(n, n-r)$ has a unique expression as $\alpha=\alpha_{i_{1}} \circ \alpha_{i_{2}} \circ$ $\cdots \circ \alpha_{i_{r}}$ with $0 \leqslant i_{1}<i_{2}<\cdots<i_{r} \leqslant n-1$, where the indices $i_{k}$ are the elements of $[n]$ such that $\left\{i_{1}, \ldots, i_{r}\right\}=\{i: \alpha(i)=\alpha(i+1)\}$. We thus can identify $S(n, n-r)$ with the set $\left\{\left(i_{r}, \ldots, i_{1}\right): 0 \leqslant i_{1}<i_{2}<\cdots<i_{r} \leqslant n-1\right\}$. In particular, the single element of $S(n, n)$, defined by the identity map on $[n]$, corresponds to the empty 0 tuple () denoted by $\emptyset_{n}$. Similarly the only element of $S(n, 0)$ is $(n-1, n-2, \ldots, 0)$. For all $n \geqslant 0$, let

$$
S(n)=\bigcup_{0 \leqslant r \leqslant n} S(n, n-r) .
$$

We say that $\alpha=\left(i_{r}, \ldots, i_{1}\right)<\beta=\left(j_{s}, \ldots, j_{1}\right)$ in $S(n)$ if $i_{1}=j_{1}, \ldots, i_{k}=j_{k}$ but $i_{k+1}>j_{k+1},(k \geqslant 0)$ or if $i_{1}=j_{1}, \ldots, i_{r}=j_{r}$ and $r<s$. This makes $S(n)$ an ordered set. 


\section{Hypercrossed Complex Pairings}

In the following we recall from [24] hypercrossed complex pairings. The fundamental idea behind these can be found in Carrasco and Ceggarra (cf. $[\mathbf{1 2}, \mathbf{1 3}]$ ). The construction depends on a variety of sources, mainly Conduché $[\mathbf{1 6}]$, Mutlu and Porter [24]. Define a set $P(n)$ consisting of pairs of elements $(\alpha, \beta)$ from $S(n)$ with $\alpha \cap \beta=\emptyset$ and $\beta<\alpha$, with respect to lexicographic ordering in $S(n)$ where $\alpha=\left(i_{r}, \ldots, i_{1}\right), \beta=\left(j_{s}, \ldots, j_{1}\right) \in S(n)$. The pairings that we will need,

$$
\left\{F_{\alpha, \beta}: N G_{n-\sharp \alpha} \times N G_{n-\sharp \beta} \rightarrow N G_{n}:(\alpha, \beta) \in P(n), n \geqslant 0\right\},
$$

are given as composites by the diagram

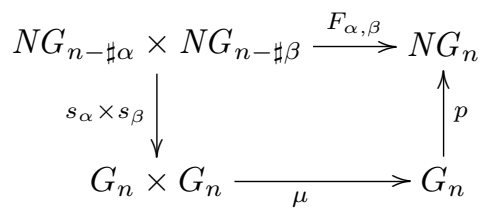

where $s_{\alpha}=s_{i_{r}}, \ldots, s_{i_{1}}: N G_{n-\sharp \alpha} \rightarrow G_{n}, s_{\beta}=s_{j_{s}}, \ldots, s_{j_{1}}: N G_{n-\sharp \beta} \rightarrow G_{n} p: G_{n} \rightarrow$ $N G_{n}$ is defined by composite projections $p(x)=p_{n-1} \ldots p_{0}(x)$, where $p_{j}(z)=$ $z s_{j} d_{j}(z)^{-1}$ with $j=0,1, \ldots, n-1 . \mu: G_{n} \times G_{n} \rightarrow G_{n}$ is given by commutator map and $\sharp \alpha$ is the number of the elements in the set of $\alpha$, similarly for $\sharp \beta$. Thus

$$
F_{\alpha, \beta}\left(x_{\alpha}, y_{\beta}\right)=p\left[s_{\alpha}\left(x_{\alpha}\right), s_{\beta}\left(x_{\beta}\right)\right] .
$$

Definition 1.2. ([24]) Let $N_{n}$, or more exactly $N_{n}^{G}$, be the normal subgroup of $G_{n}$ generated by elements of the form $F_{\alpha, \beta}\left(x_{\alpha}, y_{\beta}\right)$ where $x_{\alpha} \in N G_{n-\sharp \alpha}$ and $y_{\beta} \in$ $N G_{n-\sharp \beta}$.

This normal subgroup $N_{n}^{G}$ depends functorially on $G$, but we will usually abbreviate $N_{n}^{G}$ to $N_{n}$ when no change of group is involved.

Mutlu and Porter in [24] illustrate this normal subgroup for $n=2,3,4$, but we consider only $n=3$.

Example 1.3. For all $x_{1} \in N G_{1}, y_{2} \in N G_{2}$, the corresponding generators of $N_{3}$ are:

$$
\begin{aligned}
& F_{(1,0)(2)}\left(x_{1}, y_{2}\right)=\left[s_{1} s_{0} x_{1}, s_{2} y_{2}\right]\left[s_{2} y_{2}, s_{2} s_{0} x_{1}\right], \\
& F_{(2,0)(1)}\left(x_{1}, y_{2}\right)=\left[s_{2} s_{0} x_{1}, s_{1} y_{2}\right]\left[s_{1} y_{2}, s_{2} s_{1} x_{1}\right]\left[s_{2} s_{1} x_{1}, s_{2} y_{2}\right]\left[s_{2} y_{2}, s_{2} s_{0} x_{1}\right]
\end{aligned}
$$

and for all $x_{2} \in N G_{2}, y_{1} \in N G_{1}$,

$$
F_{(0)(2,1)}\left(x_{2}, y_{1}\right)=\left[s_{0} x_{2}, s_{2} s_{1} y_{1}\right]\left[s_{2} s_{1} y_{1}, s_{1} x_{2}\right]\left[s_{2} x_{2}, s_{2} s_{1} y_{1}\right]
$$

whilst for all $x_{2}, y_{2} \in N G_{2}$,

$$
\begin{aligned}
& F_{(0)(1)}\left(x_{2}, y_{2}\right)=\left[s_{0} x_{2}, s_{1} y_{2}\right]\left[s_{1} y_{2}, s_{1} x_{2}\right]\left[s_{2} x_{2}, s_{2} y_{2}\right] \\
& F_{(0)(2)}\left(x_{2}, y_{2}\right)=\left[s_{0} x_{2}, s_{2} y_{2}\right] \\
& F_{(1)(2)}\left(x_{2}, y_{2}\right)=\left[s_{1} x_{2}, s_{2} y_{2}\right]\left[s_{2} y_{2}, s_{2} x_{2}\right] .
\end{aligned}
$$


As a corollary, there is an equality

$$
\partial_{n}\left(N G_{n} \cap D_{n}\right)=\partial_{n}\left(N_{n}^{G} \cap D_{n}\right) .
$$

The first author and Porter [4] have shown that if $\mathbf{E}$ is a simplicial commutative algebra with Moore complex NE, and for $n>0$ the ideal generated by the degenerate elements in dimension $n$ is $E_{n}$, then

$$
\partial_{n}\left(N E_{n}\right) \supseteq \sum_{I, J} K_{I} K_{J} .
$$

This sum runs over those $\emptyset \neq I, J \subset[n-1]=\{0, \ldots, n-1\}$ with $I \cup J=[n-1]$, and $K_{I}=\bigcap_{i \in I}$ ker $d_{i}$. A similar result for simplicial Lie algebras was obtained by Akça and Arvasi in [1].

Mutlu and Porter [24] have adapted Arvasi's method to the case of simplicial groups. They gave the following result.

Proposition 1.4. ([24]) Let $\mathbf{G}$ be a simplicial group. Then for $n \geqslant 2$ and $I, J \subseteq$ $[n-1]$ with $I \cap J=[n-1]$,

$$
\left[\bigcap_{i \in I} \operatorname{ker} d_{i}, \bigcap_{j \in J} \operatorname{ker} d_{j}\right] \subseteq \partial_{n}\left(N G_{n} \cap D_{n}\right) .
$$

Castiglioni and Ladra [15] gave a general proof for the inclusions partially proved by Arvasi and Porter in [4], Arvasi and Akça in [1] and Mutlu and Porter in [24]. Their approach to the problem is different from that of the cited works. They have succeeded with a proof, for the case of algebras, over an operad by introducing a different description of the adjoint inverse of the normalization functor $\mathbf{N}: A b^{\Delta^{o p}} \rightarrow$ $C h_{\geqslant 0}$, and for the case of groups, they then adapted the construction for the adjoint inverse used for algebras to get a simplicial group $G \otimes \Lambda$ from the Moore complex of a simplicial group $G$.

The following is a result of Conduché [16].

Proposition 1.5. Let $\mathbf{G}^{\prime}$ be $(n-1)$-truncated simplicial group. Then there is a simplicial group $\mathbf{G}$ with $\operatorname{tr}_{k} \mathbf{G} \cong \mathbf{G}^{\prime}$ if and only if $\mathbf{G}^{\prime}$ satisfies the following property: For all nonempty sets of indices $(I \neq J), I, J \subset[n-1]$ with $I \cup J=[n-1]$,

$$
\left[\bigcap_{i \in I} \operatorname{ker} d_{i}, \bigcap_{j \in J} \operatorname{ker} d_{j}\right]=1 \text {. }
$$

Proof. Since $\partial_{n}\left(N G_{n}^{\prime}\right)=1$, this follows from Proposition 1.4.

\section{2-Crossed modules}

Crossed modules were introduced by Whitehead in [27]. A crossed module is a group homomorphism $\partial: M \rightarrow P$ together with an action of $P$ on $M$, written ${ }^{p} m$ for $p \in P$ and $m \in M$, satisfying the conditions $\partial\left({ }^{p} m\right)=p \partial(m) p^{-1}$ and ${ }^{\partial m} m^{\prime}=$ $m m^{\prime} m^{-1}$ for all $m, m^{\prime} \in M, p \in P$. The last condition is called the 'Peiffer identity'.

The following definition of 2-crossed module is equivalent to that given by Conduché [16]. 
A 2-crossed module of groups consists of a complex of groups

$$
L \stackrel{\partial_{2}}{\longrightarrow} M \stackrel{\partial_{1}}{\longrightarrow} N
$$

together with (a) actions of $N$ on $M$ and $L$ so that $\partial_{2}, \partial_{1}$ are morphisms of $N$ groups, and (b) an $N$-equivariant function

$$
\{\quad, \quad\}: M \times M \longrightarrow L
$$

called a Peiffer lifting. This data must satisfy the following axioms:

$$
\begin{aligned}
\text { 2CM1: } & \partial_{2}\left\{m, m^{\prime}\right\} & =\left({ }{ }_{1} m m^{\prime}\right) m m^{-1} m^{-1} \\
\text { 2CM2: } & \left\{\partial_{2} l, \partial_{2} l^{\prime}\right\} & =\left[l^{\prime}, l\right] \\
\text { 2CM3: } & (i) \quad\left\{m m^{\prime}, m^{\prime \prime}\right\} & =\partial_{1} m\left\{m^{\prime}, m^{\prime \prime}\right\}\left\{m, m^{\prime} m^{\prime \prime} m^{\prime-1}\right\} \\
& (i i) \quad\left\{m, m^{\prime} m^{\prime \prime}\right\} & =\left\{m, m^{\prime}\right\} m^{m m^{-1}}\left\{m, m^{\prime \prime}\right\} \\
\text { 2CM4: } & \left\{m, \partial_{2} l\right\}\left\{\partial_{2} l, m\right\} & =\partial_{1} m l l^{-1} \\
\text { 2CM5: } & n\left\{m, m^{\prime}\right\} & =\left\{{ }^{n} m,{ }^{n} m^{\prime}\right\}
\end{aligned}
$$

for all $l, l^{\prime} \in L, m, m^{\prime}, m^{\prime \prime} \in M$ and $n \in N$.

Here we have used ${ }^{m} l$ as a shorthand for $\left\{\partial_{2} l, m\right\} l$ in condition 2CM3 (ii) where $l$ is $\left\{m, m^{\prime \prime}\right\}$ and $m$ is $m m^{\prime}(m)^{-1}$. This gives a new action of $M$ on $L$. Using this notation, we can split 2CM4 into two pieces, the first of which is tautologous:

$$
\text { 2CM4: } \quad \begin{aligned}
& \text { (a) }\left\{\partial_{2} l, m\right\}={ }^{m} l(l)^{-1}, \\
& \text { (b) }\left\{m, \partial_{2} l\right\}=\left({ }^{2}{ }^{m} l\right)\left({ }^{m} l^{-1}\right) .
\end{aligned}
$$

The old action of $M$ on $L$, via $\partial_{1}$ and the $N$-action on $L$, is in general distinct from this second action, with $\left\{m, \partial_{2} l\right\}$ measuring the difference (by 2CM4 (b)). An easy argument using 2CM2 and 2CM4 (b) shows that with this action, ${ }^{m} l$, of $M$ on $L$, $\left(L, M, \partial_{2}\right)$ becomes a crossed module.

A morphism of 2-crossed modules can be defined in an obvious way. We thus define the category of 2-crossed modules, denoting it by $\mathbf{X}_{2} \mathbf{M o d}$.

The following theorem, in some sense, is known. We do not give the proof since it exists in the literature, $[\mathbf{1 6}],[\mathbf{2 3}]$ and $[\mathbf{2 4}, \mathbf{2 5}]$.

Theorem 1.6. The category of 2-crossed modules is equivalent to the category of simplicial groups with Moore complex of length 2.

\section{Braided, regular crossed modules and simplicial groups}

Recall that a groupoid $\mathbf{C}$ is a small category in which every arrow is an isomorphism. We write a groupoid as $\left(C_{1}, C_{0}\right)$, where $C_{0}$ is the set of objects and $C_{1}$ is the set of arrows, together with functions $s, t: C_{1} \rightarrow C_{0}$ and $e: C_{0} \rightarrow C_{1}$ such that $s e=t e=1$. The functions $s$ and $t$ are sometimes called the source and target maps, respectively. If $a, b \in C_{1}$ and $t(a)=s(b)$, then a composite $a \circ b$ exists such that $s(a \circ b)=s(a)$ and $t(a \circ b)=t(b)$. Further, this composition is associative; the elements $e_{p}, p \in C_{0}$, act as identities; and each arrow $a$ has an inverse $a^{-1}$ with $s\left(a^{-1}\right)=t(a), t\left(a^{-1}\right)=s(a), a \circ a^{-1}=e_{s(a)}$ and $a^{-1} \circ a=e_{t(a)}$.

For any groupoid $\mathbf{C}$ and $p, q \in C_{0}$, the set of arrows $a$ such that $s(a)=p$ and $t(a)=q$ is written $C_{1}(p, q)$ and termed a hom-set. If $C_{1}(p, q)$ is empty whenever $p$ 
and $q$ are distinct (that is, if $s=t$ ) then $\mathbf{C}$ is called totally disconnected. We also write $C_{1}(p, p)$ as $C_{1}(p)$. For a survey of applications of groupoids and an introduction to their literature, see [8]. In any groupoid $\left(C_{1}, C_{0}\right)$, for an element $p \in C_{0}$, the homset $C_{1}(p)$ is a group.

Now, we recall the notions of groupoid action and crossed module of groupoids from $[\mathbf{9}]$.

In the following we refer to $C_{1}$ and $C_{2}$ as the groupoids, when we of course mean $\left(C_{1}, C_{0}\right)$ and $\left(C_{2}, C_{0}\right)$.

Definition 2.1. Let $C_{1}$ and $C_{2}$ be groupoids over the same object set $C_{0}$ and let $C_{2}$ be totally disconnected. Then an action of $C_{1}$ on $C_{2}$ is given by a partially defined function

$$
C_{1} \times C_{2} \longrightarrow C_{2}
$$

written $(a, x) \mapsto x^{a}$, which satisfies:

1. $x^{a}$ is defined if and only if $t(x)=s(a)$, and then $t\left(x^{a}\right)=t(a)$,

2. $(x \circ y)^{a}=x^{a} \circ y^{a}$ and $e_{p}{ }^{a}=e_{q}$,

3. $x^{a \circ b}=\left(x^{a}\right)^{b}$ and $x^{e_{p}}=x$,

for all $x, y \in C_{2}(p)$ and $a \in C_{1}(p, q), b \in C_{1}(q, r)$.

Definition 2.2. A crossed module of groupoids consists of a pair of groupoids $C_{1}$ and $C_{2}$ over the same object set $C_{0}$, with $C_{2}$ totally disconnected, and an action of $C_{1}$ on $C_{2}$, together with a functor $\delta: C_{2} \rightarrow C_{1}$ which satisfies

CM1: $\delta\left(x^{a}\right)=a \circ(\delta x) \circ a^{-1}$

CM2: $x^{\delta y}=y \circ x \circ y^{-1}$

for all $x, y \in C_{2}(p)$ and $a \in C_{1}(p, q)$.

A crossed module of groupoids is often written diagrammatically as

$$
C: \quad C_{2} \stackrel{\delta}{\longrightarrow} C_{1} \stackrel{\stackrel{s}{\longrightarrow}}{\longrightarrow} C_{0}
$$

Note in particular that for each $p \in C_{0}, C_{2}(p) \rightarrow C_{1}(p)$ is a crossed module of groups.

Let $U$ be a monoid. A biaction of $U$ on the crossed module

$$
C: C_{2} \stackrel{\delta}{\longrightarrow} C_{1} \stackrel{s}{\underset{t}{\longrightarrow}} C_{0}
$$

consists of a pair of commuting left and right actions of $U$ on the set $C_{0}$ and on the groupoids $C_{1}$ and $C_{2}$ compatible with all of the structure. Specifically, we have functions $U \times C_{i} \rightarrow C_{i}$ and $C_{i} \times U \rightarrow C_{i}$ for $i=0,1,2$, denoted by $(u, c) \mapsto u \cdot c$ and $(c, u) \mapsto c \cdot u$ such that

BA1: each function $U \times C_{i} \rightarrow C_{i}$ determines a left action of $U$ and each function $C_{i} \times U \rightarrow C_{i}$ determines a right action of $U$ and these actions commute;

BA2: each action of $U$ preserves the groupoid structure of $C_{1}$ over $C_{0}$ and in particular the source and target maps $s, t: C_{1} \rightarrow C_{0}$ are $U$-equivariant relative to each action; 
BA3: each action of $U$ preserves the group operation in $C_{2}$ and if $x \in C_{2}(p)$ and $u \in U$ then $u \cdot x \in C_{2}(u \cdot p)$ and $x \cdot u \in C_{2}(p \cdot u)$;

BA4: each action of $U$ is compatible with the action of $C_{1}$ on $C_{2}$ so that if $x \in C_{2}(p), a \in C_{1}(p, q)$, and $u \in U$ then

$$
\begin{aligned}
& u \cdot\left(x^{a}\right)=(u \cdot x)^{u \cdot a} \in C_{2}(u \cdot q), \\
& \left(x^{a}\right) \cdot u=(x \cdot u)^{a \cdot u} \in C_{2}(q \cdot u) ;
\end{aligned}
$$

BA5: the boundary homomorphism $\delta: C_{2} \rightarrow C_{1}$ is $U$-equivariant relative to each action.

The crossed module

$$
C: C_{2} \stackrel{\delta}{\longrightarrow} C_{1} \stackrel{s}{\stackrel{s}{\longrightarrow}} C_{0}
$$

is semiregular if the object set $C_{0}$ is a monoid and there is a biaction of $C_{0}$ on $C$ in which $C_{0}$ acts on itself in its left and right regular representations. A semiregular crossed module in which $C_{0}$ is a group is said to be regular. Note that every crossed module of groups is regular.

We now recall the definition of braided regular crossed modules from [9].

Definition 2.3. A braided regular crossed module of groupoids

$$
C: C_{2} \stackrel{\delta}{\longrightarrow} C_{1} \stackrel{s}{\underset{t}{\longrightarrow}} C_{0}
$$

is a regular crossed module of groupoids with the map $\{-,-\}: C_{1} \times C_{1} \rightarrow C_{2}$, called the braiding map, satisfying the following axioms:

B1: $\{a, b\} \in C_{2}((t a)(t b)),\left\{1_{e}, b\right\}=1_{t b},\left\{a, 1_{e}\right\}=1_{t a}$ where $1_{e} \in C_{1}(e)$ is the identity arrow and $e$ is the identity element of the group $C_{0}$;

B2: $\left\{a, b \circ b^{\prime}\right\}=\{a, b\}^{t a \cdot b^{\prime}} \circ\left\{a, b^{\prime}\right\}$;

B3: $\left\{a \circ a^{\prime}, b\right\}=\left\{a^{\prime}, b\right\} \circ\{a, b\}^{a^{\prime} \cdot t b}$;

B4: $\delta\{a, b\}=(t a \cdot b)^{-1} \circ\left(a^{-1} \cdot s b\right) \circ(s a \cdot b) \circ(a \cdot t b)$;

B5: $\{a, \delta y\}=(t a \cdot y)^{-1} \circ(s a \cdot y)^{a \cdot q}$ if $y \in C_{2}(q)$;

B6: $\{\delta x, b\}=\left((x \cdot s b)^{p \cdot b}\right)^{-1} \circ(x \cdot t b)$ if $x \in C_{2}(p)$;

B7: $p \cdot\{a, b\}=\{p \cdot a, b\},\{a, b\} \cdot p=\{a, b \cdot p\},\{a \cdot p, b\}=\{a, p \cdot b\} \quad$ for all $a, a^{\prime}$, $b, b^{\prime} \in C_{1}, x, y \in C_{2}$, and $p, q \in C_{0}$.

Example 2.4. A braiding on a crossed module of groups

$$
\mathrm{C}_{2} \stackrel{\delta}{\longrightarrow} C_{1}
$$

is a function $\{-,-\}: C_{1} \times C_{1} \rightarrow C_{2}$ satisfying the following axioms:

1. $\left\{a, b b^{\prime}\right\}=\{a, b\}^{b^{\prime}}\left\{a, b^{\prime}\right\}$

2. $\left\{a a^{\prime}, b\right\}=\left\{a^{\prime}, b\right\}\{a, b\}^{a^{\prime}}$

3. $\delta\{a, b\}=[b, a]$

4. $\{a, \delta x\}=x^{-1} x^{a}$ 
5. $\{\delta y, b\}=\left(y^{-1}\right)^{b} y$, where $a, a^{\prime}, b, b^{\prime} \in C_{1}$ and $x, y \in C_{2}$.

This example leads us to define a new category. This crossed module together with the braiding map is called the braided crossed module. In fact this is a special case of a braided regular crossed module and equivalent to Conduché's reduced 2-crossed module. Brown and Gilbert (cf. [9]) have stated as a corollary that the category of braided crossed modules of groups is equivalent to that of reduced simplicial groups with Moore complex of length 2. We have proved this result by using the $F_{\alpha, \beta}$ functions in [3].

In this section, we will extend this construction to regularity. That is 'a description of the passage from a simplicial group to a braided regular crossed module'. This is a reformulation of the Brown-Gilbert result $[\mathbf{9}]$. Our aim is to show the role of the $F_{\alpha, \beta}$ functions in the structure. We will use the $F_{\alpha, \beta}$ functions in calculations of the axioms of braided regular crossed module.

Let $\mathbf{G}$ be a simplicial group with Moore complex NG. We will construct a braided regular crossed module

$$
C: C_{2} \stackrel{\delta}{\longrightarrow} C_{1} \stackrel{s}{\underset{t}{\longrightarrow}} C_{0}
$$

from the simplicial group $\mathbf{G}$.

Let $C_{0}=N G_{0}$ and $C_{1}=N G_{1} \rtimes s_{0} N G_{0}$ together with source and target maps given by $s\left(g, s_{0} p\right)=\left(d_{1} g\right) p$ and $t\left(g, s_{0} p\right)=p$ respectively. The groupoid composition in $C_{1}$ is given by

$$
\left(g_{1}, s_{0} p_{1}\right) \circ\left(g_{2}, s_{0} p_{2}\right)=\left(g_{1} g_{2}, s_{0} p_{2}\right)
$$

if $p_{1}=\left(d_{1} g_{2}\right) p_{2}$. Thus we have a groupoid $\left(C_{1}, C_{0}\right)$. Furthermore $C_{0}$ acts on the left and on the right of the groupoid $\left(C_{1}, C_{0}\right)$ by

$$
\begin{aligned}
& p \cdot\left(g, s_{0} q\right)=\left(s_{0} p g s_{0} p^{-1}, s_{0} p q\right), \\
& \left(g, s_{0} q\right) \cdot p=\left(g, s_{0} q p\right)
\end{aligned}
$$

for $p \in C_{0}$ and $\left(g, s_{0} q\right) \in C_{1}$. This action gives a biaction of $C_{0}$ on the groupoid $\left(C_{1}, C_{0}\right)$.

Now, we set $C_{2}(p)=N G_{2} / \partial_{3}\left(N G_{3} \cap D_{3}\right) \rtimes s_{1} s_{0} N G_{0} \subseteq G_{2}$ with the composition

$$
\left(l_{1}, s_{1} s_{0} p\right) \circ\left(l_{2}, s_{1} s_{0} p\right)=\left(l_{1} l_{2}, s_{1} s_{0} p\right)
$$

and the source and target maps given by

$$
s\left(l, s_{1} s_{0} p\right)=d_{1}^{1} d_{1}^{2}(l) p=p=d_{0}^{1} d_{0}^{2}(l) p=t\left(l, s_{1} s_{0} p\right) .
$$

Moreover, $C_{0}$ acts on the left and right of the groupoid $\left(C_{2}, C_{0}\right)$ by

$$
\begin{aligned}
p \cdot\left(l, s_{1} s_{0} q\right) & =\left(\left(s_{1} s_{0} p\right) l\left(s_{1} s_{0} p^{-1}\right), s_{1} s_{0} p q\right), \\
\left(l, s_{1} s_{0} q\right) \cdot p & =\left(l, s_{1} s_{0} q p\right)
\end{aligned}
$$

for $p \in C_{0}$ and $\left(l, s_{1} s_{0} q\right) \in C_{2}(q)$. Thus, we see that $p \cdot\left(l, s_{1} s_{0} q\right) \in C_{2}(p q)$ and $\left(l, s_{1} s_{0} q\right) \cdot p \in C_{2}(q p)$. This action defines a biaction of $C_{0}$ on the groupoid $\left(C_{2}, C_{0}\right)$. 
Homology, Homotopy and Applications, vol. 9(1), 2007

The action of $\left(g, s_{0} q\right) \in C_{1}$ on $\left(l, s_{1} s_{0} p\right) \in C_{2}$ can be given by

$$
\left(l, s_{1} s_{0} p\right)^{\left(g, s_{0} q\right)}=\left(\left(s_{1} g\right) l\left(s_{1} g^{-1}\right), s_{1} s_{0} q\right)
$$

if $p=\left(d_{1} g\right) q$. Define the morphism $\delta: C_{2} \rightarrow C_{1}$ by $\delta\left(l, s_{1} s_{0} p\right)=\left(d_{2} l, s_{0} p\right)$. Thus we can give the following proposition.

Proposition 2.5. The diagram

$$
C_{2} \stackrel{\delta}{\longrightarrow} C_{1} \stackrel{s}{\stackrel{s}{\longrightarrow}} C_{0}
$$

is a braided regular crossed module, where the braiding map is defined as follows:

$$
\left\{\begin{aligned}
\left\{, C_{1} \times C_{1}\right. & \longrightarrow C_{2} \\
(a, b) & \longmapsto\{a, b\},
\end{aligned}\right.
$$

for $a=\left(g_{1}, s_{0} p\right)$ and $b=\left(g_{2}, s_{0} q\right)$,

$$
\begin{aligned}
& \left\{\left(g_{1}, s_{0} p\right),\left(g_{2}, s_{0} q\right)\right\}= \\
& \left(\overline{s_{1} s_{0} p s_{1} g_{2}^{-1} s_{1} s_{0} p^{-1} s_{1} g_{1}^{-1} s_{0} g_{1} s_{1} s_{0} p s_{1} g_{2} s_{1} s_{0} p^{-1} s_{0} g_{1}^{-1} s_{1} g_{1}}, s_{1} s_{0} p q\right) \text {. }
\end{aligned}
$$

Here, on the right hand side the overline denotes a coset in $N G_{2} / \partial_{3}\left(N G_{3} \cap D_{3}\right)$ represented by an element in $N G_{2}$.

Proof. We display the elements omitting the overlines in our calculation to save complication. Firstly, we show that $\delta$ is a crossed module of groupoids by using the $F_{\alpha, \beta}$ functions.

\section{CM1:}

$$
\begin{aligned}
\delta\left(\left(l, s_{1} s_{0} p\right)^{\left(g, s_{0} q\right)}\right) & =\delta\left(\left(s_{1} g\right) l\left(s_{1} g^{-1}\right), s_{1} s_{0} q\right) \\
& =\left(d_{2}\left(\left(s_{1} g\right) l\left(s_{1} g^{-1}\right)\right), s_{0} q\right) \\
& =\left(g\left(d_{2} l\right) g^{-1}, s_{0} q\right)
\end{aligned}
$$

and

$$
\begin{aligned}
\left(g, s_{0} q\right) \circ \delta\left(l, s_{1} s_{0} p\right) \circ\left(g^{-1}, s_{0} q\right) & =\left(g, s_{0} q\right) \circ\left(d_{2} l, s_{0} p\right) \circ\left(g^{-1}, s_{0} q\right) \\
& =\left(g, s_{0} q\right) \circ\left(\left(d_{2} l\right) g^{-1}, s_{0} q\right) \\
& =\left(g\left(d_{2} l\right) g^{-1}, s_{0} q\right)
\end{aligned}
$$

Then we have

$$
\delta\left(\left(l, s_{1} s_{0} p\right)^{\left(g, s_{0} q\right)}\right)=\left(g, s_{0} q\right) \circ \delta\left(l, s_{1} s_{0} p\right) \circ\left(g^{-1}, s_{0} q\right)
$$

CM2:

$$
\begin{aligned}
\left(l, s_{1} s_{0} p\right)^{\delta\left(l^{\prime}, s_{1} s_{0} p\right)} & =\left(l, s_{1} s_{0} p\right)^{\left(d_{2} l^{\prime}, s_{0} p\right)} \\
& =\left(\left(s_{1} d_{2} l^{\prime}\right) l\left(s_{1} d_{2} l^{\prime-1}\right), s_{1} s_{0} p\right) \\
& \equiv\left(l^{\prime} l\left(l^{\prime}\right)^{-1}, s_{1} s_{0} p\right) \quad \bmod \partial_{3}\left(N G_{3} \cap D_{3}\right) \\
& =\left(\left(l^{\prime}\right), s_{1} s_{0} p\right) \circ\left(l, s_{1} s_{0} p\right) \circ\left(l^{\prime-1}, s_{1} s_{0} p\right) .
\end{aligned}
$$


Homology, Homotopy and Applications, vol. 9(1), 2007

In the calculation of axiom CM2, we have used the $F_{\alpha, \beta}$ functions. Indeed, from [24], for $l, l^{\prime} \in N G_{2}$, we have

$$
\begin{aligned}
F_{(1)(2)}\left(l^{\prime}, l\right) & =\left[s_{1} l^{\prime}, s_{2} l\right]\left[s_{2} l, s_{2} l^{\prime}\right] \\
& =s_{1} l^{\prime} s_{2} l s_{1} l^{\prime-1} s_{2} l^{\prime} s_{2} l^{-1} s_{2} l^{\prime-1} \in N G_{3} \cap D_{3},
\end{aligned}
$$

and from

$$
\partial_{3}\left(F_{(1)(2)}\left(l^{\prime}, l\right)\right)=s_{1} d_{2}\left(l^{\prime}\right) l s_{1} d_{2}\left(l^{\prime}\right)^{-1}\left(l^{\prime}\right) l^{-1}\left(l^{\prime}\right)^{-1} \in \partial_{3}\left(N G_{3} \cap D_{3}\right)
$$

we have

$$
s_{1} d_{2}\left(l^{\prime}\right) l s_{1} d_{2}\left(l^{\prime}\right)^{-1} \equiv l^{\prime} l\left(l^{\prime}\right)^{-1} \bmod \partial_{3}\left(N G_{3} \cap D_{3}\right) .
$$

Therefore the morphism $\delta$ is a crossed module of groupoids. Thus we have a regular crossed module of groupoids

$$
C: C_{2} \stackrel{\delta}{\longrightarrow} C_{1} \stackrel{s}{\stackrel{s}{\longrightarrow}} C_{0}
$$

together with the biaction of $C_{0}$ on $C$ as given above.

Now, we will show that all the axioms of a braided regular crossed module of groupoids are verified. We again use the $F_{\alpha, \beta}$ functions in the following calculations.

B1: For $a=\left(g_{1}, s_{0} p\right), b=\left(g_{2}, s_{0} q\right)$ and $1_{e}=\left(1, s_{0} e\right) ;\left\{1_{e}, b\right\}=\left(s_{1} g_{2}^{-1} s_{1} g_{2}, s_{1} s_{0} q\right)$ $=\left(1, s_{1} s_{0} q\right)=1_{t b},\left\{a, 1_{e}\right\}=\left(1, s_{1} s_{0} p\right)=1_{t a}$ and clearly $\{a, b\} \in C_{2}(p q)=$ $C_{2}((t a)(t b))$.

B2: For $a=\left(g, s_{0} p\right), b=\left(h, s_{0} q\right), b^{\prime}=\left(h^{\prime}, s_{0} q^{\prime}\right)$ it must be that

$$
\left\{a, b \circ b^{\prime}\right\}=\{a, b\}^{t a \cdot b^{\prime}} \circ\left\{a, b^{\prime}\right\},
$$

where $s\left(b^{\prime}\right)=d_{1} h^{\prime} q^{\prime}=q=t(b)$. Then,

$$
\begin{aligned}
\left\{a, b \circ b^{\prime}\right\}= & \left\{\left(g, s_{0} p\right),\left(h h^{\prime}, s_{0} q^{\prime}\right)\right\} \\
= & (\underbrace{s_{1} s_{0} p s_{1}\left(h^{\prime}\right)^{-1} s_{1} h^{-1} s_{1} s_{0} p^{-1} s_{1} g^{-1} s_{0} g s_{1} s_{0} p s_{1} h}_{A} \\
& \left.s_{1} h^{\prime} s_{1} s_{0} p^{-1} s_{0} g^{-1} s_{1} g, s_{1} s_{0}\left(p q^{\prime}\right)\right) \\
= & (\underbrace{\left(A s_{1} s_{0} p^{-1} s_{0} g^{-1} s_{1} g s_{1} s_{0} p s_{1} h^{\prime} s_{1} s_{0} p^{-1}\right)}_{B}\left(s_{1} s_{0} p s_{1}\left(h^{\prime}\right)^{-1}\right. \\
& \left.s_{1} s_{0} p^{-1} s_{1} g^{-1} s_{0} g s_{1} s_{0} p s_{1} h^{\prime} s_{1} s_{0} p^{-1} s_{0} g^{-1} s_{1} g, s_{1} s_{0} p q^{\prime}\right) \\
= & \left(A B, s_{1} s_{0} p q^{\prime}\right) \circ\left(s_{1} s_{0} p s_{1}\left(h^{\prime}\right)^{-1}\right. \\
& \left.s_{1} s_{0} p^{-1} s_{1} g^{-1} s_{0} g s_{1} s_{0} p s_{1} h^{\prime} s_{1} s_{0} p^{-1} s_{0} g^{-1} s_{1} g, s_{1} s_{0} p q^{\prime}\right) \\
= & \left(A B, s_{1} s_{0} p q^{\prime}\right) \circ\left\{a, b^{\prime}\right\},
\end{aligned}
$$


Homology, Homotopy and Applications, vol. 9(1), 2007

where

$$
\begin{aligned}
& \left(A B, s_{1} s_{0} p q^{\prime}\right)=\left(s_{1} s_{0} p s_{1}\left(h^{\prime}\right)^{-1} s_{1} h^{-1} s_{1} s_{0} p^{-1} s_{1} g^{-1} s_{0} g s_{1} s_{0} p s_{1} h s_{1} s_{0} p^{-1}\right. \\
& \left.s_{0} g^{-1} s_{1} g s_{1} s_{0} p s_{1} h^{\prime} s_{1} s_{0} p^{-1}, s_{1} s_{0} p q^{\prime}\right) \\
& =\left(s_{1} s_{0} p s_{1}\left(h^{\prime}\right)^{-1} s_{1} s_{0} p^{-1} s_{1} s_{0} p s_{1} h^{-1} s_{1} s_{0} p^{-1} s_{1} g^{-1} s_{0} g\right. \\
& \left.s_{1} s_{0} p s_{1} h s_{1} s_{0} p^{-1} s_{0} g^{-1} s_{1} g s_{1} s_{0} p s_{1} h^{\prime} s_{1} s_{0} p^{-1}, s_{1} s_{0} p q^{\prime}\right) \\
& =\left(s_{1}\left(s_{0} p\left(h^{\prime}\right)^{-1} s_{0} p^{-1}\right) s_{1} s_{0} p s_{1} h^{-1} s_{1} s_{0} p^{-1} s_{1} g^{-1} s_{0} g\right. \\
& \left.s_{1} s_{0} p s_{1} h s_{1} s_{0} p^{-1} s_{0} g^{-1} s_{1} g s_{1}\left(s_{0} p h^{\prime} s_{0} p^{-1}\right), s_{1} s_{0} p q^{\prime}\right) \\
& =\left(s_{1} s_{0} p s_{1} h^{-1} s_{1} s_{0} p^{-1} s_{1} g^{-1} s_{0} g s_{1} s_{0} p s_{1} h s_{1} s_{0} p^{-1} s_{0} g^{-1} s_{1} g\right. \text {, } \\
& \left.s_{1} s_{0} p q\right)^{\left(s_{0} p h^{\prime} s_{0} p^{-1}, s_{0} p q^{\prime}\right)} \\
& =\{a, b\}^{t a \cdot b^{\prime}}
\end{aligned}
$$

and then we have

$$
\left\{a, b \circ b^{\prime}\right\}=\{a, b\}^{t a \cdot b^{\prime}} \circ\left\{a, b^{\prime}\right\} .
$$

B3: For $a=\left(g, s_{0} p\right), a^{\prime}=\left(h, s_{0} q\right)$ and $b=\left(k, s_{0} r\right)$; (here $\left.s a^{\prime}=d_{1} h q=p=t a\right)$

$$
\begin{aligned}
& \left\{a \circ a^{\prime}, b\right\}=\left\{\left(g h, s_{0} q\right),\left(k, s_{0} r\right)\right\} \\
& =\left(s_{1} s_{0} q s_{1} k^{-1} s_{1} s_{0} q^{-1} s_{1} h^{-1} s_{1} g^{-1} s_{0} g\right. \\
& \left.s_{0} h s_{1} s_{0} q s_{1} k s_{1} s_{0} q^{-1} s_{0} h^{-1} s_{0} g^{-1} s_{1} g s_{1} h, s_{1} s_{0} q r\right) \\
& =\left(s_{1} s_{0} q s_{1} k^{-1} s_{1} s_{0} q^{-1} s_{1} h^{-1} s_{1} g^{-1} s_{0} g s_{1} s_{0} d_{1} h\right. \\
& \left.s_{1} s_{0} q s_{1} k s_{1} s_{0} q^{-1} s_{1} s_{0} d_{1} h^{-1} s_{0} g^{-1} s_{1} g s_{1} h, s_{1} s_{0} q r\right) \\
& =(\underbrace{s_{1} s_{0} q s_{1} k^{-1} s_{1} s_{0} q^{-1} s_{1} h^{-1}}_{A} s_{1} g^{-1} s_{0} g \\
& \left.s_{1} s_{0} p s_{1} k s_{1} s_{0} p^{-1} s_{0} g^{-1} s_{1} g s_{1} h, s_{1} s_{0} q r\right) \\
& =(\underbrace{A\left(s_{1} s_{0} p s_{1} k s_{1} s_{0} p^{-1} s_{1} h\right)}_{B} s_{1} h^{-1}\left(s_{1} s_{0} p s_{1} k^{-1} s_{1} s_{0} p^{-1} s_{1} g^{-1} s_{0} g\right. \\
& \left.\left.s_{1} s_{0} p s_{1} k s_{1} s_{0} p^{-1} s_{0} g^{-1} s_{1} g\right) s_{1} h, s_{1} s_{0} q r\right) \\
& =\left(B, s_{1} s_{0} q r\right) \circ\left\{\left(g, s_{0} p\right),\left(k, s_{0} r\right)\right\}^{\left(h, s_{0} q\right) \cdot r} \\
& =\left(B, s_{1} s_{0} q r\right) \circ\{a, b\}^{a^{\prime} \cdot t b},
\end{aligned}
$$

where

$$
\begin{aligned}
\left(B, s_{1} s_{0} q r\right) & =\left(s_{1} s_{0} q s_{1} k^{-1} s_{1} s_{0} q^{-1} s_{1} h^{-1} s_{1} s_{0} p s_{1} k s_{1} s_{0} p^{-1} s_{1} h, s_{1} s_{0} q r\right) \\
& \left.=s_{1} s_{0} q s_{1} k^{-1} s_{1} s_{0} q^{-1} s_{1} h^{-1} s_{0} h s_{1} s_{0} q s_{1} k s_{1} s_{0} q^{-1} s_{0} h^{-1} s_{1} h, s_{1} s_{0} q r\right) \\
& =\left\{\left(h, s_{0} q\right),\left(k, s_{0} r\right)\right\} \\
& =\left\{a^{\prime}, b\right\}
\end{aligned}
$$

since $s_{1} s_{0} p=s_{0} h s_{1} s_{0} q$. Thus we have

$$
\left\{a \circ a^{\prime}, b\right\}=\left\{a^{\prime}, b\right\} \circ\{a, b\}^{a^{\prime} \cdot t b} .
$$


Homology, Homotopy and Applications, vol. 9(1), 2007

B4:

$$
\begin{aligned}
\delta\{a, b\}= & \delta\left\{\left(g, s_{0} p\right),\left(h, s_{0} q\right)\right\} \\
= & \left(d_{2}\left(s_{1} s_{0} p s_{1} h^{-1} s_{1} s_{0} p^{-1} s_{1} g^{-1} s_{0} g s_{1} s_{0} p s_{1} h s_{1} s_{0} p^{-1} s_{0} g^{-1} s_{1} g\right), s_{1} s_{0} p q\right) \\
= & \left(s_{0} p h^{-1} s_{0} p^{-1} g^{-1} s_{0} d_{1} g s_{0} p h s_{0} p^{-1} s_{0} d_{1} g^{-1} g, s_{0} p q\right) \\
= & \left(s_{0} p h^{-1} s_{0} p^{-1}, s_{0} p q\right) \circ\left(g^{-1}, s_{0} p q\right) \circ \\
& \left(s_{0}\left(d_{1} g p\right) h s_{0}\left(d_{1} g p\right)^{-1}, s_{0} p q\right) \circ\left(g, s_{0} p q\right) \\
= & (t a \cdot b)^{-1} \circ\left(a^{-1} \cdot s b\right) \circ(s a \cdot b) \circ(a \cdot t b) .
\end{aligned}
$$

B5: For $a=\left(g, s_{0} p\right) \in C_{1}$ and $y=\left(l, s_{1} s_{0} q\right) \in C_{2}(q)$ it must be that

$$
\{a, \delta y\}=(t a \cdot y)^{-1} \circ(s a \cdot y)^{a \cdot q} .
$$

From [24], we have

$$
\partial_{3}\left(F_{(2,0)(1)}(x, t)\right)=\left[s_{0} x, s_{1} d_{2} t\right]\left[s_{1} d_{2} t, s_{1} x\right]\left[s_{1} x, t\right]\left[t, s_{0} x\right] \in \partial_{3}\left(N G_{3} \cap D_{3}\right) .
$$

Thus, for $t=p \cdot l \in N G_{2}$ and $x=g^{-1} \in N G_{1}$, we have

$$
\begin{aligned}
\{a, \delta y\}= & \left\{\left(g, s_{0} p\right),\left(d_{2} l, s_{0} q\right)\right\} \\
= & \left(s_{1} s_{0} p s_{1} d_{2} l^{-1} s_{1} s_{0} p^{-1} s_{1} g^{-1} s_{0} g s_{1} s_{0} p s_{1} d_{2} l s_{1} s_{0} p^{-1} s_{0} g^{-1} s_{1} g, s_{1} s_{0} p q\right) \\
\equiv & \left(s_{1} s_{0} p l^{-1} s_{1} s_{0} p^{-1} s_{1} g^{-1} s_{0} g s_{1} s_{0} p l s_{1} s_{0} p^{-1} s_{0} g^{-1} s_{1} g, s_{1} s_{0} p q\right) \\
& \quad \bmod \partial_{3}\left(N G_{3} \cap D_{3}\right) \\
= & \left(\left(s_{1} s_{0} p\right) l^{-1}\left(s_{1} s_{0} p\right)^{-1}, s_{1} s_{0} p q\right) \circ\left(s_{1} s_{0}\left(d_{1} g p\right) l s_{1} s_{0}\left(d_{1} g p\right)^{-1}\right. \\
& \left.s_{1} s_{0} p q\right)^{\left(g, s_{0} p q\right)} \\
= & (t a \cdot y)^{-1} \circ(s a \cdot y)^{a \cdot q} .
\end{aligned}
$$

B6: It must be that

$$
\{\delta x, b\}=\left[(x \cdot s b)^{p \cdot b}\right]^{-1} \circ(x \cdot t b) .
$$

Similarly, from $[\mathbf{2 4}]$, we have

$$
\partial_{3}\left(F_{(0)(2,1)}(k, y)\right)=\left[s_{0} d_{2} k, s_{1} y\right]\left[s_{1} k, s_{1} d_{2} x\right]\left[x, s_{1} k\right] \in \partial_{3}\left(N G_{3} \cap D_{3}\right) .
$$

Thus, for $k=l^{-1} \in N G_{2}$ and $y=p \cdot g \in N G_{1}$ we have

$$
\begin{aligned}
\{\delta x, b\}= & \left\{\delta\left(l, s_{1} s_{0} p\right),\left(g, s_{0} q\right)\right\} \\
= & \left\{\left(d_{2} l, s_{0} p\right),\left(g, s_{0} q\right)\right\} \\
= & \left(s_{1} s_{0} p s_{1} g^{-1} s_{1} s_{0} p^{-1} s_{1} d_{2} l^{-1} s_{0} d_{2} l s_{1} s_{0} p s_{1} g s_{1} s_{0} p^{-1} s_{0} d_{2} l^{-1} s_{1} d_{2} l,\right. \\
& \left.s_{1} s_{0} p q\right) \\
\equiv & \left(s_{1} s_{0} p s_{1} g^{-1} s_{1} s_{0} p^{-1} l^{-1} s_{1} s_{0} p s_{1} g s_{1} s_{0} p^{-1} l, s_{1} s_{0} p q\right) \\
& \quad \bmod \partial_{3}\left(N G_{3} \cap D_{3}\right) \\
= & \left(s_{1}\left(s_{0} p g^{-1} s_{0} p^{-1}\right) l^{-1} s_{1}\left(s_{0} p g s_{0} p^{-1}\right) l, s_{1} s_{0} p q\right) \\
= & \left(l^{-1}, s_{1} s_{0} p q\right)^{p \cdot\left(g^{-1}, s_{0} q\right)} \circ\left(l, s_{1} s_{0} p\right) \cdot q \\
= & {\left[(x \cdot s b)^{p \cdot b}\right]^{-1} \circ(x \cdot q) . }
\end{aligned}
$$


B7: For $r \in C_{0}, a=\left(g, s_{0} p\right)$ and $b=\left(h, s_{0} q\right)$

$$
\begin{aligned}
\{a \cdot r, b\}= & \left\{\left(g, s_{0} p r\right),\left(h, s_{0} q\right)\right\} \\
= & \left(s_{1} s_{0}(p r) s_{1} h^{-1} s_{1} s_{0}(p r)^{-1} s_{1} g^{-1} s_{0} g\right. \\
& \left.s_{1} s_{0}(p r) s_{1} h s_{1} s_{0}(p r)^{-1} s_{0} g^{-1} s_{1} g, s_{1} s_{0} p r q\right) \\
= & \left(s_{1} s_{0} p s_{1}\left(s_{0} r h^{-1} s_{0} r^{-1}\right) s_{1} s_{0} p^{-1} s_{1} g^{-1} s_{0} g\right. \\
& \left.s_{1} s_{0} p s_{1}\left(s_{0} r h s_{0} r^{-1}\right) s_{1} s_{0} p^{-1} s_{0} g^{-1} s_{1} g, s_{1} s_{0} p r q\right) \\
= & \left\{\left(g, s_{0} p\right),\left(s_{0} r h s_{0} r^{-1}, s_{0} r q\right)\right\} \\
= & \{a, r \cdot b\} .
\end{aligned}
$$

Furthermore, $r \cdot\{a, b\}=\{r \cdot a, b\}$ and $\{a, b\} \cdot r=\{a, b \cdot r\}$ can be shown similarly. Therefore the braided regular crossed module axioms are verified.

Remark 2.6. If the Moore complex of the simplicial group $\mathbf{G}$ is of length 2, we have $\partial_{3}\left(N G_{3} \cap D_{3}\right)=1$, and then we have a construction of a braided regular crossed module from a simplicial group with Moore complex of length 2.

Theorem 2.7. The category of braided regular crossed modules is equivalent to that of simplicial groups with Moore complex of length 2.

Proof. In the above proposition, a braided regular crossed module was already constructed by using the $F_{\alpha, \beta}$ functions from the Moore complex of a simplicial group. This defines a functor from simplicial groups to braided regular crossed modules

\section{$\Delta:$ SimpGrp $\rightarrow$ BRCM}

Conversely, we suppose that

$$
C: C_{2} \stackrel{\delta}{\longrightarrow} C_{1} \stackrel{s}{\underset{t}{\longrightarrow}} C_{0}
$$

is a braided regular crossed module. We will construct a simplicial group $\mathbf{G}$ whose Moore complex has length 2 by using the $F_{\alpha, \beta}$ functions.

Let $G_{0}=C_{0}$. The set

$$
M=\left\{a \in C_{1}: s(a)=e\right\}
$$

is a group with the following operation

$$
a b=a \circ t(a) \cdot b
$$

for $a, b \in M$. The group $C_{0}$ acts on $M$ as follows: for all $a \in M$ and $p \in C_{0}$, we set $a^{p}=p^{-1} \cdot a \cdot p$ by using the biaction of $C_{0}$ on $C_{1}$ and $C_{2}$. By using this action, we can create the semidirect product group

$$
M \rtimes C_{0}=G_{1} .
$$

The group operation in $G_{1}$ is given by

$$
(a, p)\left(a^{\prime}, p^{\prime}\right)=\left(a^{p^{\prime}} \circ\left(\left(p^{\prime}\right)^{-1} t(a) p^{\prime}\right) \cdot a^{\prime}, p p^{\prime}\right)
$$


Homology, Homotopy and Applications, vol. 9(1), 2007

for all $(a, p),\left(a^{\prime}, p^{\prime}\right) \in M \rtimes C_{0}$. Define the face and degeneracy maps by

$$
d_{0}(a, p)=p, \quad d_{1}(a, p)=p t(a), \quad s_{0}(p)=\left(0_{e}, p\right) .
$$

These maps satisfy the simplicial identities. Indeed

$$
\begin{gathered}
d_{0} s_{0}(p)=d_{0}\left(0_{e}, p\right)=p \\
d_{1} s_{0}(p)=d_{1}\left(0_{e}, p\right)=p t\left(0_{e}\right)=p e=p .
\end{gathered}
$$

Moreover, $d_{1}$ and $d_{0}$ are group homomorphisms, since

$$
\begin{array}{rlrl}
d_{1}\left((a, p)\left(a^{\prime}, p^{\prime}\right)\right) & =d_{1}\left(\left(a^{p^{\prime}} a^{\prime}, p p^{\prime}\right)\right) & d_{0}\left((a, p)\left(a^{\prime}, p^{\prime}\right)\right) & =p p^{\prime} \\
& =p p^{\prime} t\left(a^{p^{\prime}}\right) t\left(a^{\prime}\right) & & =d_{0}(a, p) d_{0}\left(a^{\prime}, p^{\prime}\right) . \\
& =p t(a) p^{\prime} t\left(a^{\prime}\right) & \\
& =d_{1}(a, p) d_{1}\left(a^{\prime}, p^{\prime}\right), &
\end{array}
$$

Furthermore, we know that $C_{2}(e)$ is a (vertex) group from [9]. An action of $a \in M$ on $y \in C_{2}(e)$ can be given by

$$
y^{a}=t(a) \cdot y \circ\{a, \delta y\}
$$

where $\{-,-\}$ is the braiding map. Then, by using this action we can create the semidirect product group $C_{2}(e) \rtimes M$.

Moreover, an action $(a, p) \in M \rtimes C_{0}$ on $\left(y, a^{\prime}\right) \in C_{2}(e) \rtimes M$ can be given by

$$
\begin{aligned}
\left(y, a^{\prime}\right)^{\left(0_{e}, p\right)} & =\left(y^{p},\left(a^{\prime}\right)^{p}\right) \\
\left(y, a^{\prime}\right)^{(a, e)} & =\left(t(a) \cdot y \circ\{a, \delta y\}, a^{a^{\prime}}\right) .
\end{aligned}
$$

Using this action, we have the semidirect product

$$
G_{2}=\left(C_{2}(e) \rtimes M\right) \rtimes\left(M \rtimes C_{0}\right)
$$

and homomorphisms

$$
\begin{aligned}
d_{0}\left(y, a, a^{\prime}, p\right) & =\left(a^{\prime}, p\right) \\
d_{1}\left(y, a, a^{\prime}, p\right) & =\left(a \circ t(a) \cdot a^{\prime}, p\right)=\left(a a^{\prime}, p\right) \\
d_{2}\left(y, a, a^{\prime}, p\right) & =\left(\delta(y) \circ t(\delta(y)) \cdot a, p t\left(a^{\prime}\right)\right)=\left(\delta(y) a, p t\left(a^{\prime}\right)\right) \\
s_{0}\left(a^{\prime}, p\right) & =\left(0_{e}, 0_{e}, a^{\prime}, p\right) \\
s_{1}(a, p) & =\left(0_{e}, a, 0_{e}, p\right) .
\end{aligned}
$$

We thus have a 2-truncated simplicial group $\left\{G_{2}, G_{1}, G_{0}\right\}$ that looks like

$$
\left(C_{2}(e) \rtimes M\right) \rtimes\left(M \rtimes C_{0}\right) \underset{s_{0}^{1}, s_{1}^{1}}{\stackrel{d_{0}^{2}, d_{1}^{2}, d_{2}^{2}}{\rightleftarrows}}\left(M \rtimes C_{0}\right) \stackrel{d_{0}^{1}, d_{1}^{1}}{\rightleftarrows} C_{0} .
$$

There is a $\mathbf{C o s k}_{2}$ functor from the category of 2-truncated simplicial groups to that of simplicial groups. We set $\mathbf{G}^{\prime}=\operatorname{Cosk}_{2}\left\{G_{2}, G_{1}, G_{0}\right\}$ and claim $N G_{3}^{\prime}=1$. We now give the sketch of the argument. From the result of Conduché as given in 
Lemma 1.1 of this paper, for the 2-truncated simplicial group $\left\{G_{2}, G_{1}, G_{0}\right\}$, we have $N\left(\mathbf{C o s k}_{2}\left\{G_{2}, G_{1}, G_{0}\right\}\right)_{3}=1$. From [24], by using the image of $F_{\alpha, \beta}$ functions, we have that $\partial_{3}\left(N G_{3} \cap D_{3}\right)$ is the product of $\left[\operatorname{ker} d_{2}, \operatorname{ker} d_{0} \cap \operatorname{ker} d_{1}\right]$, [ker $d_{1}, \operatorname{ker} d_{0} \cap$ $\operatorname{ker} d_{2}$ ], [ker $\left.d_{0}, \operatorname{ker} d_{1} \cap \operatorname{ker} d_{2}\right]$, [ $\left[\operatorname{ker} d_{0} \cap \operatorname{ker} d_{2}, \operatorname{ker} d_{0} \cap \operatorname{ker} d_{1}\right]$, [ker $d_{1} \cap \operatorname{ker} d_{2}, \operatorname{ker} d_{0}$ $\left.\cap \operatorname{ker} d_{1}\right]$ and [ker $d_{1} \cap \operatorname{ker} d_{2}$, ker $d_{0} \cap \operatorname{ker} d_{2}$ ]. A direct calculation using the descriptions of the face maps and the actions above shows that these are all trivial, so $\partial_{3}\left(N G_{3} \cap D_{3}\right)=1$, but again $\partial_{3}$ is a monomorphism so $N G_{3}^{\prime}=1$ as required.

\section{Crossed Squares and Braided, Regular Crossed Modules}

Loday and Guin-Walery $[\mathbf{2 6}]$ introduced the notion of crossed square as an algebraic model of connected 3-types.

A crossed square of groups is a commutative square of groups;

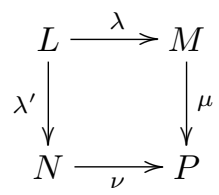

together with left actions of $P$ on $L, M, N$ and a function $h: M \times N \rightarrow L$. Let $M$ and $N$ act on $M, N$ and $L$ via $P$. The structure must satisfy the following axioms for all $l \in L, m, m^{\prime} \in M, n, n^{\prime} \in N, p \in P$;

(i) the homomorphisms $\mu, \nu, \lambda, \lambda^{\prime}$ and $\mu \lambda$ are crossed modules and both $\lambda, \lambda^{\prime}$ are $P$-equivariant,

(ii) $h\left(m m^{\prime}, n\right)=h\left({ }^{m} m^{\prime},{ }^{m} n\right) h(m, n)$,

(iii) $h\left(m, n n^{\prime}\right)=h(m, n) h\left({ }^{n} m,{ }^{n} n^{\prime}\right)$,

(iv) $\lambda h(m, n)=m^{n} m^{-1}$

(v) $\lambda^{\prime} h(m, n)={ }^{m} n n^{-1}$,

(vi) $h(\lambda l, n)=l^{n} l^{-1}$,

(vii) $h\left(m, \lambda^{\prime} l\right)={ }^{m} l l^{-1}$,

(viii) $h\left({ }^{p} m,{ }^{p} n\right)={ }^{p} h(m, n)$.

Conduché constructed (private communication to Brown in 1984; see also published version $[\mathbf{1 7}]$ ) a 2 -crossed module from a crossed square

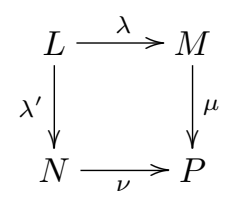

as

$$
L \stackrel{\left(\lambda^{-1}, \lambda^{\prime}\right)}{\longrightarrow} M \rtimes N \stackrel{\mu \nu}{\longrightarrow} P .
$$

In [5], by taking the bisimplicial nerve of the crossed square and using the ArtinMazur codiagonal functor (cf. [2]), we obtained a 2-truncated simplicial group $\mathbf{G}^{(2)}$ 
Homology, Homotopy and Applications, vol. 9(1), 2007

that looks like

$$
\mathbf{G}^{(2)}:(L \rtimes(N \rtimes M)) \rtimes(N \rtimes(M \rtimes P)) \underset{s_{0}^{1}, s_{1}^{1}}{\stackrel{d_{0}^{2}, d_{1}^{2}, d_{2}^{2}}{\rightleftarrows}} N \rtimes(M \rtimes P) \frac{d_{0}^{1}, d_{1}^{1}}{\rightleftarrows} P
$$

with the faces and degeneracies as given in [5]. We also showed that the Moore complex of this 2-truncated simplicial group

$$
N G_{2} \stackrel{\partial_{2}}{\longrightarrow} N G_{1} \stackrel{\partial_{1}}{\longrightarrow} N G_{0}
$$

is isomorphic to the mapping cone complex

$$
L \stackrel{\left(\lambda^{-1}, \lambda^{\prime}\right)}{\longrightarrow} M \rtimes N \stackrel{\mu \nu \quad \longrightarrow}{\longrightarrow} P
$$

of the crossed square, and that this mapping cone has a 2-crossed module structure.

In this section, we will construct a braided regular crossed module as

$$
C: C_{2} \stackrel{\delta}{\longrightarrow} C_{1} \stackrel{s}{\underset{t}{\longrightarrow}} C_{0}
$$

by applying the Brown-Gilbert functor from 2-crossed modules to braided regular crossed modules to this mapping cone complex (1).

Clearly we have $C_{0}=P \cong G_{0}^{(2)}$, and by using the action of $P$ on $M \rtimes N$, we have $C_{1}=(M \rtimes N) \rtimes P \cong N G_{1}^{(2)} \rtimes s_{0}\left(G_{0}^{(2)}\right)$. The source and target maps are given by $s(m, n, p)=\mu(m) \nu(n) p$ and $t(m, n, p)=p$ for $m \in M, n \in N, p \in P$. The groupoid composition in $C_{1}$ is given by

$$
(m, n, p) \circ\left(m^{\prime}, n^{\prime}, p^{\prime}\right)=\left(m^{\nu(n)} m^{\prime}, n n^{\prime}, p^{\prime}\right)
$$

if $\mu\left(m^{\prime}\right) \nu\left(n^{\prime}\right) p^{\prime}=p$. We have

$$
t\left((m, n, p) \circ\left(m^{\prime}, n^{\prime}, p^{\prime}\right)\right)=p^{\prime}=t\left(m^{\prime}, n^{\prime}, p^{\prime}\right)
$$

and

$$
\begin{aligned}
s\left((m, n, p) \circ\left(m^{\prime}, n^{\prime}, p^{\prime}\right)\right) & =s\left(\left(m^{\nu(n)} m^{\prime}, n n^{\prime}, p^{\prime}\right)\right) \\
& =\mu\left(m^{\nu(n)} m^{\prime}\right) \nu(n) \nu\left(n^{\prime}\right) p^{\prime} \\
& =\mu(m) \nu(n) \mu\left(m^{\prime}\right) \nu\left(n^{\prime}\right) p^{\prime} \\
& =\mu(m) \nu(n) p \\
& =s(m, n, p),
\end{aligned}
$$

and thus we have a groupoid structure $\left(C_{1}, C_{0}\right)$. The biaction of the group $C_{0}$ on the groupoid $\left(C_{1}, C_{0}\right)$ can be given by

$$
\begin{aligned}
p \cdot(m, n, q) & =\left({ }^{p} m,{ }^{p} n, p q\right) \\
(m, n, q) \cdot p & =(m, n, q p)
\end{aligned}
$$

for $(m, n, q) \in C_{1}$ and $p \in C_{0}$. 
Homology, Homotopy and Applications, vol. 9(1), 2007

We also have $C_{2}=L \rtimes P \cong N G_{2}^{(2)} \rtimes s_{0} s_{0}\left(N G_{0}^{(2)}\right)$, and the groupoid composition can be given by

$$
(l, p) \circ\left(l^{\prime}, p\right)=\left(l l^{\prime}, p\right),
$$

and the biaction of $C_{0}$ on the groupoid $\left(C_{2}, C_{0}\right)$ can be given by

$$
p \cdot(l, q)=\left({ }^{p} l, p q\right), \quad(l, q) \cdot p=(l, q p) .
$$

Thus we have a regular crossed module

$$
C: L \rtimes P \stackrel{\delta^{\prime}}{\longrightarrow}(M \rtimes N) \rtimes P \underset{t}{\stackrel{s}{\rightleftarrows}} P
$$

from the mapping cone complex $(1)$, where the morphism $\delta^{\prime}$ is given by $(l, p) \mapsto$ $\left(\lambda^{-1}(l), \lambda^{\prime}(l), p\right)$. The braiding map on this structure is given by

$$
\left\{(m, n, p),\left(m^{\prime}, n^{\prime}, p^{\prime}\right)\right\}=\left(h\left({ }^{\nu\left(n^{-1}\right)} m^{-1}, n^{-1}\left({ }^{p} m^{\prime}\right) n\right), p p^{\prime}\right)
$$

for $m, m^{\prime} \in M, n, n^{\prime} \in N$ and $p, p^{\prime} \in P$, where $h$ is the $h$-map of the crossed square.

Thus, if given a crossed square

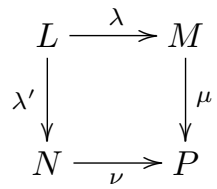

its associated braided regular crossed module is

$$
L \rtimes P \stackrel{\delta^{\prime}}{\longrightarrow}(M \rtimes N) \rtimes P \stackrel{s}{\stackrel{t}{\longrightarrow}} P
$$

as described above.

Now, let

$$
C: C_{2} \stackrel{\delta}{\longrightarrow} C_{1} \stackrel{s}{\underset{t}{\longrightarrow}} C_{0}
$$

be any braided regular crossed module. Consider its associated 2-truncated simplicial group

$$
\mathbf{G}^{\prime}:\left(C_{2}(e) \rtimes M\right) \rtimes\left(M \rtimes C_{0}\right) \underset{s_{0}^{1}, s_{1}^{1}}{\stackrel{d_{0}^{2}, d_{1}^{2}, d_{2}^{2}}{\rightleftarrows}}\left(M \rtimes C_{0}\right) \stackrel{d_{0}^{1}, d_{1}^{1}}{\rightleftarrows} C_{s_{0}^{0}} \underset{\gtrless}{\rightleftarrows}
$$

together with the face and degeneracy maps as given in Theorem 2.7.

We investigate the Moore complex of this 2-truncated simplicial group. Clearly $N G_{0}^{\prime}=C_{0}$. By the definition of $d_{0}^{1}$, we have ker $d_{0}^{1}=N G_{1}^{\prime}=M$, and by the definition of $d_{1}^{1}$, we have

$$
\text { ker } d_{1}^{1}=\bar{M}=\left\{(m, p): p=t(m)^{-1}, m \in M, p \in P\right\} .
$$

Similarly, by the definition of the face maps $d_{0}^{2}$ and $d_{1}^{2}$, we have ker $d_{0}^{2} \cap \operatorname{ker} d_{1}^{2}=$ $N G_{2}^{\prime}=C_{2}(e)$. 
Homology, Homotopy and Applications, vol. 9(1), 2007

Mutlu and Porter in [24] defined a functor from simplicial groups to crossed squares, denoting it by

$$
\mathbf{M}(-, 2): \text { SimpGrp } \rightarrow \mathbf{C r s}^{2} .
$$

We now briefly explain this functor.

Let $\mathbf{G}$ be a simplicial group. Then the following diagram

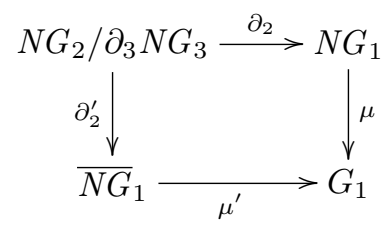

is the underlying square of a crossed square. The extra structure is given as follows; $N G_{1}=\operatorname{ker} d_{0}^{1}$ and $\overline{N G}_{1}=\operatorname{ker} d_{1}^{1}$. Since $G_{1}$ acts on $N G_{2} / \partial_{3} N G_{3}, \overline{N G}_{1}$ and $N G_{1}$, there are actions of $\overline{N G}_{1}$ on $N G_{2} / \partial_{3} N G_{3}$ and $N G_{1}$ via $\mu^{\prime}$, and $N G_{1}$ acts on $N G_{2} / \partial_{3} N G_{3}$ and $\overline{N G}_{1}$ via $\mu$. Both $\mu$ and $\mu^{\prime}$ are inclusions, and all actions are given by conjugation. The $h$-map is

$$
\begin{aligned}
h: N G_{1} \times \overline{N G}_{1} & \longrightarrow N G_{2} / \partial_{3} N G_{3} \\
(x, \bar{y}) & \longmapsto h(x, y)=\left[s_{1} x, s_{1} y s_{0} y^{-1}\right] \partial_{3} N G_{3} .
\end{aligned}
$$

Here $x$ and $y$ are in $N G_{1}$ as there is a bijection between $N G_{1}$ and $\overline{N G}_{1}$.

Now, we apply this functor to the 2-truncated simplicial group $\mathbf{G}^{\prime}$ given above. From Section 2, we have $\partial_{3}\left(N G_{3}^{\prime}\right)=1$. In the above calculations, we have $N G_{0}^{\prime}=C_{0}$, $N G_{1}^{\prime}=M, \overline{N G_{1}^{\prime}}=\bar{M}$ and $N G_{2}^{\prime}=C_{2}(e)$. Thus we have a crossed square $\mathbf{M}\left(\mathbf{G}^{\prime}, 2\right)$ that looks like

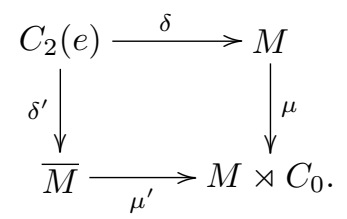

The braiding map $\{-,-\}: C_{1} \times C_{1} \rightarrow C_{2}$ induces the $h$-map of the crossed square.

Thus, if given a braided regular crossed module

$$
C: C_{2} \stackrel{\delta}{\longrightarrow} C_{1} \stackrel{s}{\stackrel{s}{\longrightarrow}} C_{0}
$$

its associated crossed square is

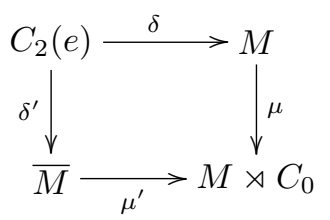

as described above. 
Homology, Homotopy and Applications, vol. 9(1), 2007

\section{Quadratic modules from braided regular crossed modules}

Quadratic modules of groups were initially defined by Baues in $[6]$ as models for connected 3-types. In [5, Section 5], we constructed a functor from 2-crossed modules to quadratic modules. Furthermore, in [5], by using the $F_{\alpha, \beta}$ functions, we gave a construction of a quadratic module from a simplicial group.

Recall that a nil(2)-module is a pre-crossed module $\partial: M \rightarrow N$ with an additional "nilpotency" condition. This condition is $P_{3}(\partial)=1$, where $P_{3}(\partial)$ is the subgroup of $M$ generated by Peiffer commutator $\left\langle x_{1}, x_{2}, x_{3}\right\rangle$ of length 3 .

The Peiffer commutator in a pre-crossed module $\partial: M \rightarrow N$ is defined by

$$
\langle x, y\rangle=\left({ }^{\partial x} y\right) x y^{-1} x^{-1}
$$

for $x, y \in M$.

For a group $G$, the group

$$
G^{a b}=G /[G, G]
$$

is the abelianisation of $G$ and

$$
\partial^{c r}: M^{c r}=M / P_{2}(\partial) \rightarrow N
$$

is the crossed module associated to the pre-crossed module $\partial: M \rightarrow N$. Here $P_{2}(\partial)=\langle M, M\rangle$ is the Peiffer subgroup of $M$.

The following definition is due to Baues (cf. [6]).

Definition 4.1. A quadratic module $(\omega, \delta, \partial)$ is a diagram

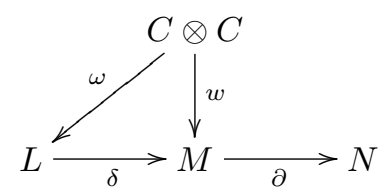

of homomorphisms between groups such that the following axioms are satisfied.

QM1: The homomorphism $\partial: M \rightarrow N$ is a nil(2)-module with Peiffer commutator map $w$ defined above. The quotient map $M \rightarrow C=\left(M^{c r}\right)^{a b}$ is given by $x \mapsto$ $\bar{x}$, where $\bar{x} \in C$ denotes the class represented by $x \in M$ and $C=\left(M^{c r}\right)^{a b}$ is the abelianisation of the associated crossed module $M^{c r} \rightarrow N$.

QM2: The boundary homomorphisms $\partial$ and $\delta$ satisfy $\partial \delta=1$ and the quadratic map $\omega$ is a lift of the Peiffer commutator map $w$, that is $\delta \omega=w$ or equivalently

$$
\delta \omega(\bar{x} \otimes \bar{y})=\left({ }^{\partial x} y\right) x y^{-1} x^{-1}=\langle x, y\rangle
$$

for $x, y \in M$.

QM3: $L$ is an $N$-group and all homomorphisms of the diagram are equivariant with respect to the action of $N$. Moreover, the action of $N$ on $L$ satisfies the formula $(a \in L, x \in M)$

$$
{ }^{2 x} a=\omega((\bar{x} \otimes \overline{\delta a})(\overline{\delta a} \otimes \bar{x})) a .
$$


Homology, Homotopy and Applications, vol. 9(1), 2007

QM4: Commutators in $L$ satisfy the formula $(a, b \in L)$

$$
\omega(\overline{\delta a} \otimes \overline{\delta b})=[b, a] .
$$

Now, consider the braided regular crossed module

$$
C: C_{2} \stackrel{\delta}{\longrightarrow} C_{1} \stackrel{s}{\underset{t}{\longrightarrow}} C_{0}
$$

and its associated 2-crossed module

$$
C_{2}(e) \stackrel{\delta}{\longrightarrow} M \stackrel{t}{\longrightarrow} C_{0}
$$

as given in [9]. Applying the functor from 2-crossed modules to quadratic modules as described in [5, Section 5] to this associated 2-crossed module (2) gives a quadratic module

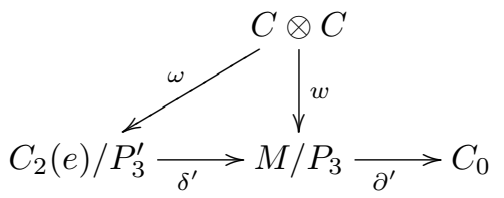

where $P_{3}$ is the normal subgroup of $M$ generated by elements of the form

$$
\langle x,\langle y, z\rangle\rangle \text { and }\langle\langle x, y\rangle, z\rangle
$$

for $x, y, z \in M$. The Peiffer elements in $M$ are given by $\langle x, y\rangle=\left({ }^{t(x)} y\right) x y^{-1} x^{-1}$ for $x, y \in M$. Also, $P_{3}^{\prime}$ is the normal subgroup of $C_{2}(e)$ generated by elements of the form

$$
\{x,\langle y, z\rangle\} \text { and }\{\langle x, y\rangle, z\}
$$

where $\{-,-\}$ is the Peiffer map of the associated 2-crossed module (2).

Since $t(\langle x,\langle y, z\rangle\rangle)=1$ and $t(\langle\langle x, y\rangle, z\rangle)=1$, the map $\partial^{\prime}: M / P_{3} \rightarrow C_{0}$ given by $\partial^{\prime}\left(m P_{3}\right)=t(m)$ is a well defined group homomorphism. In addition, since $\delta\{x,\langle y, z\rangle\}=\langle x,\langle y, z\rangle\rangle$ and $\delta\{\langle x, y\rangle, z\}=\langle\langle x, y\rangle, z\rangle$, the map $\delta^{\prime}: C_{2}(e) / P_{3}^{\prime} \rightarrow M / P_{3}$ given by $\delta^{\prime}\left(x P_{3}^{\prime}\right)=\delta(x) P_{3}$ is a well defined group homomorphism. The braiding map induces the quadratic map $\omega$.

Thus, if given a braided regular crossed module

$$
C: C_{2} \stackrel{\delta}{\longrightarrow} C_{1} \underset{t}{\stackrel{s}{\longrightarrow}} C_{0},
$$

its associated quadratic module is

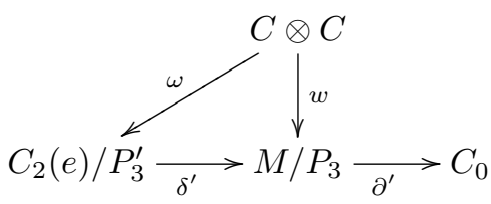

as described above. 
Homology, Homotopy and Applications, vol. 9(1), 2007

\section{References}

[1] I. AkÇa And Z. Arvasi, Simplicial and crossed lie algebras, Homology, Homotopy Appl., 4(1), 43-57, (2002).

[2] M. Artin and B. Mazur, On the Van Kampen theorem, Topology 5, 179189, (1966).

[3] Z. Arvasi, M. Koçak and E. Ulualan, Braided crossed modules and reduced simplicial groups, Taiwanese J. Math. 9(3), 477-488, (2005).

[4] Z. Arvasi and T. Porter, Higher dimensional Peiffer elements in simplicial commutative algebras, Theory Appl. Categ. 3(1), 1-23, (1997).

[5] Z. Arvasi and E. Ulualan, On algebraic models for homotopy 3-types, Journal of Homotopy and Related Structures 1(1), 1-27, (2006).

[6] H.J. Baues, Combinatorial Homotopy and 4-Dimensional Complexes, Walter de Gruyter, Berlin, De Gruyter expositions in mathematics, (1991).

[7] H.J. Baues, Algebraic Homotopy, Cambridge University Press, Cambridge, (1989).

[8] R. Brown, From groups to groupoids, Bull. London Math. Soc. 19, 113-134, (1987).

[9] R. Brown and N.D. Gilbert, Algebraic models of 3-types and automorphism structures for crossed modules, Proc. London Math. Soc. (3), 59, 5173, (1989).

[10] R. BRown And J-L. Loday, Van Kampen theorems for diagram of spaces, Topology 26, 311-335, (1987).

[11] R. Brown and C.B. Spencer, G-groupoids, crossed modules and the fundamental groupoid of topological group, Proc. Konink. Nederl. Akad. Wetensch. 79, 296-302, (1976).

[12] P. Carrasco, Complejos hipercruzados cohomologia y extensiones, Ph.D. thesis, Universidad de Granada, (1987).

[13] P. Carrasco and A.M. Cegarra, Group-theoretic algebraic models for homotopy types, J. Pure Appl. Algebra 75, 195-235, (1991).

[14] P. Carrasco and A.M. Cegarra, Braided tensor structures on homotopy groupoids and nerves of (braided) categorical groups, Comm. Algebra 24(3), 3995-4058, (1996).

[15] J.L. Castiglioni and M. Ladra, Peiffer elements in simplicial groups and algebras, math.KT/0501260 v1, (2005).

[16] D. Conduché, Modules croisés généralisés de longueur 2, J. Pure Appl. Algebra 34, 155-178, (1984).

[17] D. Conduché, Simplicial crossed modules and mapping cones, Georgian Math. J. 10(4), 623-636, (2003).

[18] J. Duskin, Simplicial Methods and the Interpretation of Triple Cohomology, Memoirs of the A.M.S. 163, (1975).

[19] G.J. Ellis, Crossed squares and combinatorial homotopy, Math. Z. 214, 93-110, (1993). 
[20] A.R. Garzon and J.G. Miranda, Homotopy theory for (braided) catgroups, Cahiers Topologie Géom. Différentielle Catég. XXXVIII-2, (1997).

[21] A. Joyal and R. Street, Braided monoidal categories, Macquarie Mathematics Report 860081, Macquarie University, (1986).

[22] D.M. KAN, A combinatorial definition of homotopy groups, Annal. Math. 61, 288-312, (1958).

[23] J.L. Loday, Spaces with finitely many non-trivial homotopy groups, J. Pure Appl. Algebra 24, 179-202, (1982).

[24] A. Mutlu And T. Porter, Applications of Peiffer pairings in the Moore complex of simplicial group, Theory Appl. Categ. 4(7), 148-173, (1998).

[25] A. Mutlu And T. Porter, Freeness conditions for 2-crossed modules and complexes, Theory Appl. Categ. 4(8), 174-194, (1998).

[26] D. Guin-Walery and J.L. Loday, Obstruction á l'excision en K-théories algébrique, In: Friedlander, E.M., Stein, M.R. (eds.) Evanston Conf. on Algebraic K-Theory 1980. (Lect. Notes Math. 854, 179-216) Springer, Berlin, Heidelberg, New York (1981).

[27] J.H.C. Whitehead, Combinatorial homotopy I and II, Bull. Amer. Math. Soc. 55, 231-245 and 453-496, (1949).

Z. Arvasi zarvasi@ogu.edu.tr

Mathematics Department

Eskişehir Osmangazi University

Eskişehir 26480

Turkey

E. Ulualan eulualan@dumlupinar.edu.tr

Mathematics Department

Dumlupınar University

Kütahya

Turkey

This article is available at http://intlpress.com/HHA/v9/n1/a5 